\title{
Exploring Social Practices of Peer-Review in an Agent-Based Simulation: The COST Action PEERE
}

\author{
$\underline{\text { Virginia Dignum }}^{\text {a }}$ and Frank Dignum ${ }^{\text {b }}$

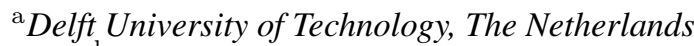 \\ ${ }^{\mathrm{b}}$ Utrecht University, The Netherlands \\ Email: m.v.dignum@tudelft.nl
}

\begin{abstract}
The peer-review process was designed to assure the validity and quality of science that seeks publication. As such, peer review is a cornerstone of science, confirming the "worth" of scientific studies and results. However, the peer-review process is increasingly seen as biased, opaque, slow and inconsistent. Its quality and efficiency depends on a complex, large-scale collaboration process, which is sensitive to motivations, incentives and institutional contexts.

PEERE (New Frontiers of Peer Review), a recent EU-funded action, aims to improve efficiency, transparency and accountability of peer review through a trans-disciplinary, cross-sectorial collaboration. In this paper, we report on the results of one of PEERE's activities, in which we explore, in a simulated environment, different approaches to peer reviewing. The objectives of this Action are: (i) to analyse peer review in different scientific areas by integrating quantitative and qualitative research and incorporating recent experimental and computational findings, (ii) to evaluate the implications of different models of peer review and to explore new incentive structures, rules and measures to improve collaboration in all stages of the peer review process; (iii) to involve science stakeholders in data sharing and testing initiatives, and, (iv) to collaboratively define a joint research agenda that points to an evidence-based peer review reform.

In order to understand the effect of different peer-review models on the behaviour of reviewers, an understanding of the context and culture of peer review is required. In this paper, we present a framework to analyse different peer-review formats, based on models of social aspects of human motivation. In order to analyse the effect of different peer-review models on the behaviour of reviewers, a deep understanding of the context and culture of peer review is required, which includes a rich model of agent's motives and behaviours. The theory of human motivation by McClelland distinguishes four motives (McClelland (1987)):
\end{abstract}

- Achievement: is about achieving goal states and drives people to try to achieve different things, thus, fostering explorative behaviour that satisfies the need for novelty.

- Power: is about trying to have an impact on the world, and includes both the need to control both its own and other's actions.

- Affiliation: drives people to seek the company of others and to establish and maintain positive interactions with others.

- Avoidance: leads to self preservation, seeking certainty, and emotional regulation, and fosters the categorization and simplification of behaviour so that it becomes more standardized (and thus predictable).

Moreover, our simulation framework supports the specification of different peer-review practices. These practices are then compared by letting agents with different social frames enact the different reviewing approaches.

The model considers motives and social practices as basis for deliberation. We show initial simulation results on two different peer-review processes.

Keywords: Peer review, social practice theory, agent-based modelling and simulation 


\section{INTRODUCTION}

Peer review is a cornerstone of science, whose quality and efficiency depends on a complex, large-scale collaboration process, which is sensitive to motivations, incentives and institutional contexts. Peer review is therefore one of the main issues that makes science a complex social system. It is applied to many spheres of scientific activity such as funding, publication, recruitment and even research productivity evaluation (Squazzoni and Takács (2011)). It provides quality assurance and improves scientific knowledge through expert opinions. However, the current process of peer review is not without its flaws. Peer review has been subject to intense criticism for slowing down the publication process, bias against specific categories of paper and author, unreliability, inability to detect errors and fraud, unethical practices, and the lack of recognition for reviewers (Walker and Rocha da Silva (2015); Smith (2006, 2009); War (2011)). In fact, peer-review is often compared to democracy: a system full of problems but better than any alternative yet we have. Despite the many different approaches to peer-review, little is still known about their effectiveness and appropriateness to the reviewing process (Smith (2006)).

Recent proofs of the failures of peer review, resulting from judgement bias and parochialism, and cases of misconduct, have lead to an increasing call for the reconsideration of the rigour and quality of the process. Moreover, there is an increasing concern that the increase on the number of submissions, due to publishing pressures related to tenure and academic evaluation, and to the growing participation of new countries in the scientific process, will render the traditional reviewing model impossible to maintain.

The EU funded COST Action PEERE (www.peere.org) aims to improve efficiency, transparency and accountability of peer review through trans-disciplinary and cross-sectorial collaboration. In this paper, we mostly focus on PEERE's objective of evaluating the implications of different models of peer review, and exploring new incentive structures, rules and measures to improve collaboration in all stages of the peer review process. To this effect, we propose a simulation environment to analyse different peer review models.

\section{Models of Peer Review}

In this section, we shortly introduce some of the innovative forms of peer review that are currently gaining significance in their attempts to solve (some of) these problems. A comprehensive, recent, survey of novel peer-review approaches can be found in (Walker and Rocha da Silva (2015)).

- Collective or Cross-peer Review: Rather than having referees individually review a paper and send their reports back to the journal editor, referees are encouraged to evaluate and discuss each other's review reports with the intent to minimize any potential errors and bias in referees' reports, and help referees give a more balanced evaluation; e.g. used in many peer-reviewed scientific conferences and is now a possibility for Science publications (http://www.sciencemag.org/) and for Nature's The EMBO Journal (http://www.nature.com/emboj).

- interactive: After an initial independent peer review, real-time collaboration between authors and reviewers is encouraged until consensus is reached; e.g. used in Frontiers (http://www.frontiersin.org/). The author rebuttal process used in many conferences is also a type of interactive review.

- transparent: where peer-review comments are included alongside published papers, possibly indicating the identity of the reviewer(s); used often in combination with post-publication peer review.

- post-publication: after a quick internal check all articles deemed appropriate are published under a label "awaiting peer review", upon publication the review process is started; increasingly used by many journals as option, and by publishers as F1000Research (http://f1000research.com/), PlosONE (http://www.plosone.org/) and PeerJ (https://peerj.com/).

Finally, the rapidly expanding role of preprint servers (e.g., ArXiv: http://arxiv.org/) that dispense with traditional peer review altogether (Walker and Rocha da Silva (2015)). Preprint servers enable authors to by-pass the delays, biases, unreliability and restrictions associated with classic peer review, by publishing their work directly online. The main impact of preprint servers has been in physics, mathematics, and astronomy, but also other disciplines are increasingly relying on such online repositories as a means of dissemination of scientific thought. For example, the RePEc (Research Papers in Economics) repository aims to disseminate research in Economics and related sciences, containing over 1.7 million research pieces from 2,100 journals and 4,000 working paper series, including working papers, journal articles, books, book chapters and software components. 


\section{The Game of Peer-Review}

In a broad sense, peer-review can be seen as a strategic game, where players (i.e. authors, reviewers, and journals/editors) seek to increase their own position while ensuring a global high level of quality. However, in the scientific process of peer-review, each actor is both an author and a reviewer. So, actors will base their decisions on their appreciation of the situation from both perspectives. At a very abstract level, we can describe the peer-review process from two perspectives: that from the overall scientific progress, and that of a self-interested player.

From the perspective of scientific advance, peer-review is assumed to determine the quality of a paper, which when published will contribute to the advance of the scientific field and possibly have an impact on science and society. Peer-review can help authors to improve their papers and help maintain the standards of any given journal. Journals are therefore interested in attracting the most qualified reviewers to ensure the quality of accepted papers. One might argue that the publishers of the journal also have a stake in the process, but if we assume that high quality journals get better cited and thus have more readership and generate more income for them, then the incentive of profit and high scientific quality are aligned and the journals can be seen to represent the role of independent scientific advance.

From the perspective of a self-interested player, at any given time a player can choose between three different strategies (Leek et al. (2011)): (1) write and submit a paper, (2) review a paper and reject, or (3) review a paper and accept. The first strategy has the potential to improve a player's status, by increasing its number of publications. If a player chooses either of the first two strategies, it can ensure that the status of other actors will not increase. However, choosing strategy (3), the status of another player will potentially increase. In the classical type of peer-review, authors will not know who the reviewer is, which means that a reviewer can choose for strategies (2) and (3) without needing to take into account the effect on its social relationship with the authors, which, in the case of double-blind reviews are not even known to the reviewer. However, in open review settings, the identity of the reviewer will be made known to the authors and to the whole research field. In this case, the self-interested strategy described above, will not suffice as the reviewer will need to consider also the social consequences of its decisions.

Putting the two perspectives together, can be seen as a case of Prisoner's Dilemma. In the most simple version rewards can be seen as follows, illustrated in Table 3. When a high-quality paper is accepted by a journal, both the journal and the author increase their status (i.e. reward=1 for both). Journals loose status if a bad quality paper is accepted for publication (i.e. journal is punished: reward=-1). Authors always benefit from paper acceptance (i.e. reward=1). Authors will always loose when their paper is rejected, as they've put time and effort in its writing (i.e. punishment: reward=-1). Given the above table where the rewards and punishments

Table 1. The publishing dilemma: rewards for authors and journals

\begin{tabular}{|c|c|c|c|}
\hline \multicolumn{2}{|c|}{} & \multicolumn{2}{c|}{ Journal } \\
\cline { 3 - 4 } \multicolumn{2}{|c|}{} & Accept & Reject \\
\hline \multirow{2}{*}{ Author } & Good paper & $(1,1)$ & $(-1,-1)$ \\
\cline { 2 - 4 } & Bad Paper & $(1,-1)$ & $(-1,1)$ \\
\hline
\end{tabular}

are all equal a journal should accept good papers and reject bad papers. However, the situation changes if the journal receives many submissions. In this case the rejection of a good paper is not so bad (because another good paper might take its place and the overall quality of the journal is still good). In that case whenever the journal is in doubt whether a paper is good or bad it will reject the paper, because the punishment for publishing a bad paper is worse than rejecting a good paper.

Unfortunately, from a modelling viewpoint, real life is even more complex than the situation depicted above. As illustrated in the self-interested perspective, when acting the reviewer's role, actors are seldom solely motivated by the interests of the journal, but may consider how the acceptance or rejection of the paper they are reviewing affects their own position. A reviewed paper might support their own work or just pre-empt the publication of their own research. Moreover, the publication of a bad quality paper can negatively reflect on the status of the reviewer as an author in the same journal, and therefore influence the author's publication strategy. 
The above discussion shows that understanding peer-review will require more complex models than those based on actors' utilities. In the next section, we will introduce a model to analyse peer review that is based on current Social Science thought on human motivations and relations.

\section{Social Aspects of Peer-Review}

In order to analyse the effect of different peer-review models on the behaviour of reviewers, a deep understanding of the context and culture of peer review is required, which includes a rich model of agent's motives and behaviours. Human behaviour results from a need to balance between novelty and control. I.e. in the one hand, seeking out new situations, while, on the other hand, trying to avoid uncertainty and, thus, strive to predict and control the environment. However, this does not readily indicate how people choose concrete actions, or more specifically, how researchers decide to agree to review and consequently decide on acceptance or rejection of that submission. We provide an initial model of agents, representing reviewers, using our social intelligent system architecture (?), which includes: a) Motives, based on McClelland's theory of basic human motivations (McClelland (1987)) Motives provide a balance between approach and avoidance mechanisms, and can account for the effect of incentives in reviewers behaviour b) Social Identity, to describe the way people position themselves, and others, in terms of membership of, possibly many, social groups (i.e. reference groups) and social goals are often based on comparison with others (Tajfel (1974)). c) External vs. internal drives, to understand and consider internal (pro-active) drives and external (reactive) drives in reviewer choice processes. d) Social Practices, allowing to cope with the complexity of combining social reality and behaviour in such an environment, we use the concept of social practice (Reckwitz (2002)). Social practices are conditioned behaviours that result from past interactions.

\subsection{Human Motives}

In the current version of the framework, we focus of the modelling of human motivations (McClelland (1987)) in order to get more concrete handles on drivers of behaviour. McClelland argues that there are a number of basic natural incentives that give rise to some motives. Besides the biological (homeostatic) motives such as hunger and need for sleep (which are, in fact, not very salient in most of the social situations), McClelland distinguishes four motives: Achievement, Power, Affiliation and Avoidance.

Taking human motives as starting point has a number of important consequences for modelling the peerreview process. Motives provide a balance between approach and avoidance mechanisms. E.g. where the power motive can lead one to agree on reviewing tasks for the possibility this gives to exert power over others, the affiliation motive makes sure that this is not done at all costs, and not always, but, specially in the case of open-reviewing processes, is kept within "socially acceptable" bounds. In the same way, the achievement motive leads people to explore new ways to achieve goals, and therefore to choose for performing research and writing papers over agreeing to review others' papers, while, the avoidance motive ensures that one does what is expected in the situation and one avoids getting rejections by putting a lot of effort in writing a paper. An agent model based on such motives supports flexible and situational deliberation in dynamic contexts of changing peer-reviewing approaches.

Besides the motives some social concepts like social identity, values and norms play a role in the interactions and decisions people make (see e.g. (Dignum et al. (2014))). However, in this paper we want to focus on the concept of social practice as a practical way to encompass some of the other aspects and as a starting point for more complex models.

\subsection{Social Practices}

Social practices are patterns of activity meaningful to people as parts of their everyday life, and the way these are typically and habitually performed in (much of) a society, e.g. going to work, cooking, or showering. In social science, social practices are formulated by some researchers as taking the centre stage to the extent that people, and things, occupy secondary roles as the carriers of practice (Shove et al. (2012)). In our framework agents identify suitable social practices given a situation and take those as heuristics for appropriate behaviour in that situation. Researchers in social science (Holtz (2014); Reckwitz (2002)) have identified three broad categories of elements of social practices: Material: covers all physical aspects of the performance of a practice, including the human body and the actions that can be performed as part of the social practice. This relates to our physical aspects of a situation; Meaning: refers to the issues which are considered to be (socially) relevant with respect to that material, i.e. understandings, beliefs and emotions. This relates to our social aspects of a situation; and, Competence: refers to skills and knowledge which are required to perform the practice. This 
relates to our notion of deliberation about a situation.

One could see a social practice as an elaborate condition-action rule. First, a situation is assessed in order to check whether a social practice is relevant. When it is relevant all material elements get a social interpretation (partly) determined by the social practice. Thus, a group of people can be given meaning as being "my colleagues", "a 'rival' research team" or "publishers", depending on the social practice that is activated. Within a social practice several behaviours are possible. Depending on the competence that a person has, she will disregard some. Then an action is chosen for which a kind of standard social effect is also expected within this social practice.

Individuals and societies typically evolve a collection of practices over time that can be adopted in different situations. Social practices are like social norms in that they emerge from individuals, but are not dependent on the individuals any more. They are continuously shaped when they are followed and can differ for individuals with different experiences. E.g. we all share an understanding of the greeting practice, but the exact behaviours and social connotations may differ. Moreover, depending on the situation, the personality and the skills of an individual, carrying out a practice will be a more automatic or a more deliberated process.

\section{Peer-review Simulation Framework}

\begin{tabular}{|c|c|c|c|}
\hline Social practice & $\begin{array}{l}\text { Classical Peer-review } \\
\text { (double-blind) }\end{array}$ & $\begin{array}{l}\text { Open Post-publication Peer- } \\
\text { review }\end{array}$ & Glance review \\
\hline $\begin{array}{l}\text { Purpose } \\
\text { Physical } \\
\text { Social }\end{array}$ & \multicolumn{3}{|c|}{$\begin{array}{l}\text { Paper publishing } \\
\text { Ensure scientific quality; maintain journal standards }\end{array}$} \\
\hline $\begin{array}{r}\text { Physical Context } \\
\text { Resources } \\
\text { Places } \\
\text { Actors }\end{array}$ & \multicolumn{3}{|l|}{$\begin{array}{l}\text { Submissions; reviews } \\
--- \\
\text { Reviewers, Authors, Editors }\end{array}$} \\
\hline Activities & \multicolumn{3}{|c|}{ Paper submission; publishing; informative acceptance; acceptance; superficial reading;rejection; } \\
\hline $\begin{array}{r}\text { Social Context } \\
\text { Social } \\
\text { interpretation } \\
\text { Social attitude } \\
\text { Norms }\end{array}$ & $\begin{array}{l}\text { Authors and reviewers unknown } \\
\text { to each other; } \\
\text { Impartiality; remove bias; ... } \\
\text { Anonymity } \\
\text { Editor does not reveal identities, } \\
\text { not even after publication; } \\
\text { Reviewer should provide } \\
\text { informative review; } \\
\text { Reviewer can forward paper to be } \\
\text { reviewed by sub-reviewer (junior } \\
\text { colleague, student) }\end{array}$ & $\begin{array}{l}\text { Authors and reviewers are known } \\
\text { to each other; } \\
\text { Increase accountability; } \\
\text { Openness } \\
\text { Collaboration between authors } \\
\text { and reviewers towards } \\
\text { improvement of paper } \\
\text { Positive criticism is expected }\end{array}$ & $\begin{array}{l}\text { Authors and reviewers unknown } \\
\text { to each other; } \\
\text { Avoid bias; spread of opinions; } \\
\text { ease reviewer's task } \\
\text { Anonymity } \\
\text { Wisdom of the crowd; } \\
\text { Permitted to give short "yes/no" } \\
\text { review; } \\
\text { Reviewer cannot use sub- } \\
\text { reviewers }\end{array}$ \\
\hline Plan patterns & $\begin{array}{l}\text { Reviewer accepts request; } \\
\text { Review performed (possibly by } \\
\text { sub-reviewer); } \\
\begin{array}{ll}\text { If acceptance } & \text { Then } \\
& \text { paper revised; } \\
& \text { next review; } \\
\text { Else } & \text { publishing } \\
\text { paper rejected }\end{array}\end{array}$ & $\begin{array}{l}\text { Paper published as 'under review'; } \\
\text { Review performed; } \\
\text { Interaction between reviewer and } \\
\text { author for revised paper; } \\
\text { If approval Then } \\
\begin{array}{l}\text { Else } \quad \text { status "Reviewed" } \\
\text { paper retracted }\end{array}\end{array}$ & $\begin{array}{l}\text { Many reviewers invited to review; } \\
\text { Reviewers accept; } \\
\text { Review decision taken quickly; } \\
\text { If acceptance Then } \\
\text { one thorough review } \\
\text { Else } \quad \text { paper rejected }\end{array}$ \\
\hline Meaning & $\begin{array}{l}\text { Competitive behaviour; fulfilment } \\
\text { of power motive for reviewer }\end{array}$ & $\begin{array}{l}\text { Cooperative behaviour; fulfilment } \\
\text { of affiliation motive for reviewer }\end{array}$ & $\begin{array}{l}\text { supports the avoidance motive for } \\
\text { reviewer }\end{array}$ \\
\hline Competences & Subject knowledge & $\begin{array}{l}\text { Subject knowledge } \\
\text { Cooperative behaviour }\end{array}$ & $\begin{array}{l}\text { Subject knowledge; } \\
\text { large reviewing experience }\end{array}$ \\
\hline
\end{tabular}

Figure 1. Peer-Review as Social Practice: comparison of peer-review approaches.

The framework implements our model of social deliberation based on social practices (Dignum and Dignum (2015)). The components of this representation model are illustrated in Figure 1, describing the differences between classical, open and glance peer-review approaches. Depending on their motives and social identities, agents will apply these practices for different reasons and satisfying different aims. E.g. under a double-blind 
reviewing schema, a reviewer can satisfy its power motive by using its knowledge to reject papers of others, whereas under an open reviewing schema, the power motive is satisfied by the possibility to look smart and objective in public, by the fact that you are the one that can decide on the acceptance of the paper. Moreover, in case the paper is from a very well-known researcher, a power-motivated reviewer will tend to accept that paper because of the power relation. Concerning the affiliation motive, reviewers are able to use an open reviewing schema to establish and enforce their relations with research peers, possibly resulting in a stronger network. In the classical review schema, the possibility to improve its relation with the editor or program chair, will satisfy a reviewer's affiliation motive. As for the case of the glance review, reviewers are expected to quickly decide on the merit of a paper, assuming their vast past reviewing experience. In this case, the amount of work for the reviewer is minimal, but through the wisdom of the crowd, decisions on the paper are close to the classical case. It can satisfies the avoidance motive of reviewers because it gives them the possibility to participate on the scientific process without too much effort.

\subsection{Initial Experiments and Discussion}

It is well-known that, in the classical reviewing format, it is increasingly difficult for editors to find suitable reviewers. Without entering here into details, this can be partly attributed to the fact that researchers do not see a direct contribution of their reviewing tasks to the satisfaction of their desires and motives. Nevertheless, most researchers are motivated to do some effort to their community.

In this initial experiment, we compare the results of classical peer-review with a so-called glance-review approach. In our simulation model, each agent is characterized by its level of reviewing expertise, which determines the quality of their review, and by its probability of error. Papers are generated with a given level of quality, which agents with high expertise are more likely to approach. Journals can follow either a classic peer-review or a glance review process. In both cases, journals aim at accepting papers of high quality. This is achieved by the review process in which reviews are as close as possible to the given paper quality. In this experiment, we aim to show that paper quality can be approximated by glance-reviews given a large enough population of glance-reviewers.

For the experiment, a population of 100 reviewers was generated, each with a given expertise level, and 1000 papers were generated with random quality between 0 and 100. For each paper, 3 reviewers were randomly selected to perform a classic peer-review (in the following called formal-reviewers) and other 50 reviewers were selected to perform a glance-review (in the following called glance-reviewers). We generated formalreviewers by a Normal distribution around expertise level 75 (maximum expertise is 100), with ranges of $10 \%$ and $20 \%$. The review score of a formal-reviewer is calculated by a normal distribution around the paper quality, with deviation based on the reviewers expertise. The reviewer score of a glance-reviewer is based on a Poisson distribution for top and bottom quality papers, and is random for the mid-section. Glance-reviewers have a precision level, indicating the proportion of papers the reviewer will consider to be in the top and bottom range. We used $10 \%$ and $20 \%$ precision levels.

Overall simulation results, based on 1000 papers, are given in Figure 2. It shows that formal-reviewers closely approximate the paper quality in both deviation conditions. As for the glance-reviewers, in the $10 \%$ precision case (i.e. where reviewers are not very qualified), reviews are quite far for paper quality, which affects the acceptance of papers by the journal. This however should not be a problem if one reasons from the motives of reviewers. In fact, in small scientific communities, the affiliation motive will be higher and therefore, journals are more likely to be able to attract reviewers for classical review process. In larger communities, affiliation plays a lesser role, and avoidance (of extra work) is more usual. However, in these communities journals will be able to use a glance-review approach as the likelihood of higher precision is high. Furthermore, an independent-samples t-test was conducted to compare the review scores in the classical peer-review condition and the 'random'-review conditions. There was no significant difference in the review scores for the classical $(\mathrm{M}=47.4, \mathrm{SD}=4.53)$ and random $(\mathrm{M}=44.9, \mathrm{SD}=6.75)$ conditions; $\mathrm{t}(191)=0.97, \mathrm{p}=0.45$. These results suggest that taking a sufficient large number of 'random' reviews approximates quite closely the scores of traditional reviews by 3 expert reviewers.

\section{Conclusions}

In this paper, we argue that the process of peer-review is influenced by many social aspects that have to be taken into account when investigating and comparing different alternative processes. We subsequently show the first results of a simulation platform that we are developing and discuss the results of an agent-based simulation of reviewer's behaviour under different peer-review models as described above. Agents were built according 


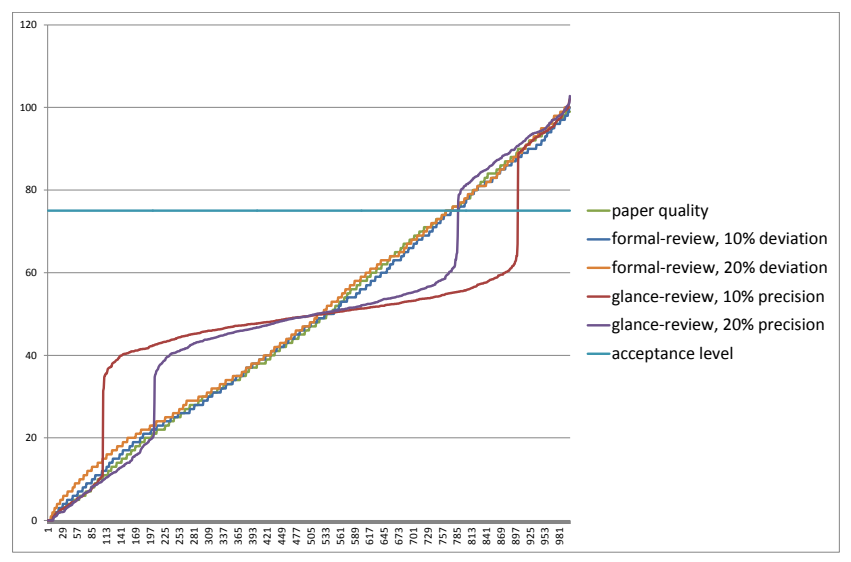

Figure 2. Simulation results.

to the Social Intelligent System Architecture. At each simulation step, agents can decide on different options concerning their scientific career: accepting reviewer tasks, performing own research, collaborate with others, etc. The simulation is then used to analyse the effect of different peer-review models on the network and reputation of agents and on the complexity and amount of reviewing processes. Of course we have shown only a first step and plan to extend the platform and make it available for the research community to experiment with all kinds of different peer-review processes in a realistic way. This work contributes to the objectives of the COST Action PEERE by providing a platform to model, compare and analyse different peer-review approaches from the perspective of their contribution to the motives and social interactions of the reviewers. Only by understanding how researchers perceive peer-review and how it fits on their common practices, can we deliberate about the suitability and acceptability of new forms of peer-review.

Acknowledgements. This research is supported by COST action TD1306: PEERE (www.peere.org).

\section{REFERENCES}

Dignum, F. and Dignum, V. (2015). Contextualized planning using social practices. In Coordination Organisation Institutions and Norms, volume LNAI 9372. Springer.

Dignum, F., Prada, R., and Hofstede, G. (2014). From autistic to social agents. In AAMAS 2014.

Holtz, G. (2014). Generating social practices. JASSS, 17(1):17.

Leek, J. T., Taub, M. A., and Pineda, F. J. (2011). Cooperation between referees and authors increases peer review accuracy. PLoS ONE, 6(11):e26895.

McClelland, D. (1987). Human Motivation. Cambridge Univ. Press.

Reckwitz, A. (2002). Toward a theory of social practices. Eur. J. Social Theory, 5(2):243-263.

Shove, E., Pantzar, M., and Watson, M. (2012). The Dynamics of Social Practice. Sage.

Smith, R. (2006). Peer review: a flawed process at the heart of science and journals. Journal of the Royal Society of Medicine, 99(4):178-182.

Smith, R. (2009). In search of an optimal peer review system. Journal of Participatory Medicine, 1(e13).

Squazzoni, F. and Takács, K. (2011). Social simulation that 'peers into peer review'. Journal of Artificial Societies and Social Simulation, 14(4):3.

Tajfel, H. (1974). Social identity and intergroup behavior. Social Science Information, 13(2):65-93.

Walker, R. and Rocha da Silva, P. (2015). Emerging trends in peer reviewa survey. Frontiers in Neuroscience, (9):169.

War, M. (2011). Peer review: Recent experience and future directions. New Review of Information Networking, 16(1):23-53. 